\title{
Power law decay of local density of states oscillations near a line defect in a system with semi-Dirac points
}

\author{
Wang Chen, Xianzhe Zhu, Xiaoying Zhou, ${ }^{*}$ and Guanghui Zhou \\ Department of Physics, Key Laboratory for Low-Dimensional Structures and Quantum \\ Manipulation (Ministry of Education), Hunan Normal University, Changsha 410081, China
}

\begin{abstract}
We theoretically study the power-law decay behavior of the local density of states (LDOS) oscillations near a line defect in system with semi-Dirac points by using a low-energy $k \cdot p$ Hamiltonian. We find that the LDOS oscillations are strongly anisotropic and sensitively depend on the orientation of the line defect. We analytically obtain the decay indexes of the LDOS oscillations near a line defect running along different directions by using the stationary phase approximation. Specifically, when the line defect is perpendicular to the linear dispersion direction, the decay index is $-5 / 4$ whereas it becomes $-1 / 4$ if the system is gapped, both of which are different from the decay index $-3 / 2$ in isotropic Dirac systems. In contrast, when the line defect is perpendicular to the parabolic dispersion direction, the decay index is always $-1 / 2$ regardless of whether the system is gapped or not, which is the same as that in a conventional semimetal. In general, when the defect runs along an arbitrary direction, the decay index sensitively depends on the incident energy for a certain orientation of the line defect. It varies from $-5 / 4$ to $-1 / 2$ due to the absence of strict stationary phase point. Our results indicate that the decay index $-5 / 4$ provides a fingerprint to identify semi-Dirac points in $2 \mathrm{D}$ electron systems.
\end{abstract}

\section{INTRODUCTION}

The discovery of graphene has triggered a boom of study on the Dirac-Weyl fermions in condensed matter systems on account of both rich physics therein and promising applications [1,2]. Graphene possesses a gapless energy spectrum with linear dispersion around two inequivalent Dirac points in its Brillouin zone [2]. This peculiar band structure contributes to unique transport properties such as Klein tunneling [3] and half-integer quantum-Hall effect [4]. When a graphene is subjected to anisotropic strain, the nearest hoppings also become anisotropic, and the Dirac points will move towards each other [5-9]. Under critical anisotropy, two inequivalent Dirac points merge into a semi-Dirac point (SDP), around which the energy dispersion is linear in one direction and parabolic in the perpendicular direction [7-9].

Besides strained graphene, SDP in energy spectrum has also been predicted in many other systems, such as the strained or electric field modulated few-layer black phosphorus [10-15], multilayer $\left(\mathrm{TiO}_{2}\right)_{\mathrm{n}} /\left(\mathrm{VO}_{2}\right)_{\mathrm{m}}$ nanostructures [16-18], silicene oxide [19], $\mathrm{Bi}_{1-x} \mathrm{Sb}_{x}$ thin film [20], striped boron sheet [21], strained monolayer arsenene [22] and spiral multiferroic oxide modulated surface states in topological insulators $[23,24]$. To date, semi-Dirac spectrum has been observed experimentally in potassium doped few-layer black phosphorus [10], tunable ultracold atomic honeycomb optical lattice [25], and polariton honeycomb lattices [26]. Although the dispersion around a SDP is a combination of that in conventional semimetals and Dirac materials, the low-energy physics in it may exhibit unique features which can't be fully understood by combing the existed results such as the unusual Landau levels [7, 8, 17, 27], optical conductivity [28, 29], anisotropic plasmon [30], and Fano factor in ballistic transport [31].

*Electronic address: xiaoyingzhou@hunnu.edu.cn
Impurities and defects in materials induce many interesting physical phenomena, such as the quasi-particle interference (QPI) patterns [33-36] and the RKKY interaction between magnetic defect lines [32] in graphene. QPI induced by line defects or point impurities gives an oscillation pattern of the local density of states (LDOS) in the vicinity of the imperfections [33-36]. Those LDOS oscillations can be directly probed using the scanning tunneling microscope [3438]. The wave vector corresponding to the QPI pattern depends on the geometry of the constant energy contour (CEC) [37]. Hence, the relevant properties of Fermi surface can be extracted from the LDOS, which makes the QPI image is particularly useful in probing the dispersion of the surface bands [34-38]. In turn, the QPI patterns exhibit unique characteristics in different electron systems [36, 38, 39]. In system with isotropic Dirac points such as graphene or the surface states of three dimensional (3D) topological insulators, the power-law decay behavior of LDOS near a line defect is $x^{-3 / 2}[38,39]$, which is much faster than $x^{-1 / 2}$ in conventional two-dimensional (2D) electron gas [36], where $x$ is the distance away from the line defect. These decay indexes serve as fingerprints to characterize related physical systems $[36,38,39]$. Since the low-energy dispersion around SDP is inherited from that in conventional semimetals and isotropic Dirac materials, a natural question is what is the power-law decay index of the LDOS oscillations near a line defect in electron system with SDPs?

Herein, this work studies the power-law decay behavior of the LDOS oscillations near a line defect in a 2D semiDirac system. The line defect is modeled by an ultrathin high rectangular barrier, which is also adopted in previous works [39-41] studying the LDOS oscillation near it on the surface of 3D topological insulators. Using a low-energy $k \cdot p$ Hamiltonian, we find that the LDOS oscillations are strongly anisotropic, sensitively depending on the orientation of the defect. We analytically obtain the decay indexes of the LDOS oscillations in various cases by using stationary phase approximation [39-42]. Specifically, when the line defect is perpendicular to the linear dispersion direction, the decay 
index of the LDOS is $-5 / 4$ whereas the it becomes $-1 / 4$ if the SDP is gapped, both of which are different from the index $-3 / 2$ in system with isotropic Dirac points. However, when the line defect is perpendicular to the parabolic dispersion direction, the decay indexes are $-1 / 2$ regardless of the SDP is gapped or not, which is the same as that in a conventional semimetal. Further, when the line defect is perpendicular to an arbitrary direction between the linear and parabolic dispersion directions, the decay index sensitively depends on the incident energy due to the absence of strict stationary phase point. It varies from $-5 / 4$ to $-1 / 2$. Our results indicate that the powerlaw decay index $-5 / 4$ provides a fingerprint to verify the SDP in $2 \mathrm{D}$ electron systems.

The rest of this paper is organized as follows. In Sec. II, we introduce the low-energy effective model and the stationary phase approximation. Sec. III presents some numerical results and discusses of the LDOS oscillations in various cases combined with analytical analysis based on the stationary phase approximation. In Sec. IV, we summarize our work.

\section{MODEL AND METHOD}

The effective low-energy Hamiltonian around a semi-Dirac point is $[7,11]$

$$
H=\frac{\hbar^{2} k_{y}^{2}}{2 m^{*}} \sigma_{x}+\hbar v_{F} k_{x} \sigma_{y}+\Delta \sigma_{z},
$$

where $\sigma_{x}, \sigma_{y}$ and $\sigma_{z}$ are the Pauli matrices, $m^{*}$ the effective mass and $v_{F}$ the Fermi velocity, and $\mathbf{k}=\left(k_{x}, k_{y}\right)$ the wavevector. We also include a gap $\Delta$ in Hamiltonian (1) to explore whether it impacts the LDOS oscillation or not. Typically, the two parameters in potassium doped few-layer black phosphorus [11] are $v_{F}=3 \times 10^{5} \mathrm{~m} / \mathrm{s}$ and $m^{*}=$ $1.42 m_{e}$, where $m_{e}$ is the free electron mass. According to the data of the angular resolved photoelectron spectroscopy measurement in Ref [10], Hamiltonian (1) is valid in the energy regime within $0.4 \mathrm{eV}$ relative to the semi-Dirac point. The corresponding eigenvalue is

$$
E_{ \pm}\left(k_{x}, k_{y}\right)= \pm \sqrt{\frac{\hbar^{4} k_{y}^{4}}{4 m^{* 2}}+\hbar^{2} v_{F}^{2} k_{x}^{2}+\Delta^{2}},
$$

where $E_{+/-}$is the conduction/valence band. The eigenvector is

$$
\Psi_{\mathbf{k}}(\mathbf{r})=\left(\begin{array}{c}
1 \\
\chi
\end{array}\right) e^{i \mathbf{k} \cdot \mathbf{r}}
$$

with $\chi=\left(\hbar^{2} k_{y}^{2} / 2 m^{*}+i \hbar v_{F} k_{x}\right) /(E+\Delta)$. For $\Delta=0$, Eq. (2) is the energy dispersion near a SDP. As shown in Fig. 1(c), the energy band linearly (parabolically) disperses along the $k_{x}\left(k_{y}\right)$ direction. Fig. 1(d) depicts the density of states (DOS) corresponding to Fig. 1(c). In contrast to the linearly dependent DOS around the isotropic Dirac point [2], the DOS around the SDP is proportional to $E^{1 / 2}[17,30]$.

Following previous works studying the LDOS oscillations near a line defect on the surface of $3 \mathrm{D}$ topological insulators

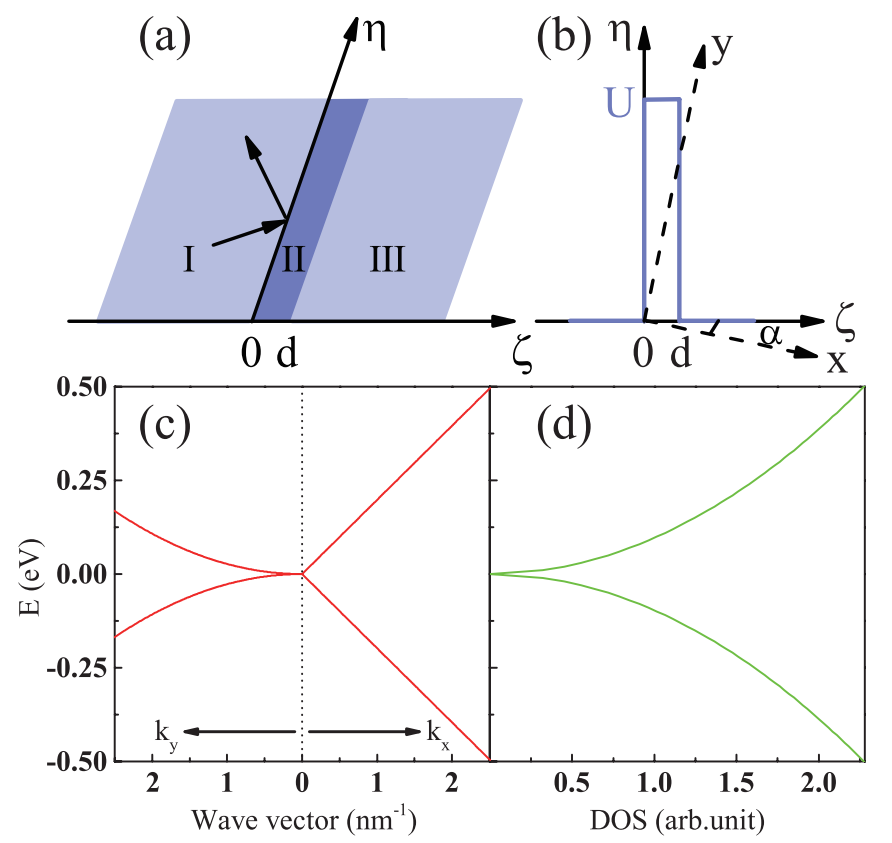

FIG. 1: (a) Schematic diagram of the scattering problem. (b) The profile of the electric potential. (c) Band structure and (d) density of states near a 2D semi-Dirac point.

[39-41], we model the line defect as a ultrathin rectangular electric barrier [see Figs. 1(b)]. The limitation of this barrier is a $\delta$-potential if we keep $U d \equiv$ constant with decreasing width $(d \rightarrow 0)$. We also study the LDOS oscillations near the line defect by modeling it as a $\delta$-potential in the Appendix. Without loss of generality, we assume that the barrier is parallel (perpendicular) to the $\eta(\zeta)$ direction at angle $\alpha \in$ $[0, \pi / 2]$ with respect to the $x$-axis [see Fig. 1(b)]. Where $\alpha=0 / \frac{\pi}{2}$ corresponds to the line defect perpendicular to the linear/parabolic dispersion direction. The potential profile is $U(\zeta)=U_{0}[\Theta(\zeta)-\Theta(\zeta-d)]$ with $\Theta(\cdot)$ the Heaviside step function. Then, the wave vectors $k_{x}$ and $k_{y}$ can be transformed in terms of $k_{\zeta}$ and $k_{\eta}$ [30], i.e., $k_{x}=k_{\zeta} \cos \alpha-k_{\eta} \sin \alpha$ and $k_{y}=k_{\zeta} \sin \alpha+k_{\eta} \cos \alpha$.

As shown in Fig. 1(a), the scattering frame is divided into three regions, i.e., the incident region I, the barrier region II, and the transmitted region III. Owing to the translation invariance in the $\eta$-direction, the transverse wave vector $k_{\eta}$ is a good quantum number. Therefore, the wave function admits the form $\Psi_{\mathbf{k}}(\zeta, \eta)=e^{i k_{\eta} \eta} \varphi(\zeta)$. The scattered wave is characterized by the longitudinal wave vector $k_{\zeta}$ result from the energy conservation. For briefness, we express all quantities in dimensionless units by introducing a length unit $l_{0}=1 \mathrm{~nm}$ and an energy unit $E_{0}=\hbar^{2} /\left(2 m^{*} l_{0}^{2}\right)=26.8 \mathrm{meV}$. Hereafter, the lengths (energies) are in unit of $l_{0}\left(E_{0}\right)$, and the wavevectors are in unit of $1 / l_{0}$ throughout the paper. For certain $k_{\eta}$ and $E$, the longitudinal wave vectors $k_{\zeta}$ of each region are governed by

$$
(E-U)^{2}=\Delta^{2}+\left[h_{1}\left(k_{\zeta}, k_{\eta}\right)\right]^{4}+u^{2}\left[h_{2}\left(k_{\zeta}, k_{\eta}\right)\right]^{2},
$$

where $h_{1}\left(k_{\zeta}, k_{\eta}\right)=k_{\zeta} \sin \alpha+k_{\eta} \cos \alpha, h_{2}\left(k_{\zeta}, k_{\eta}\right)=k_{\zeta} \cos \alpha-$ 
$k_{\eta} \sin \alpha$, and the dimensionless quantities $u=2 m^{*} v_{F} l_{0} / \hbar$. This is a quartic algebraic equation about the wave vector $k_{\zeta}$ except for the case of $\alpha=0$. For $\alpha \neq 0$, the wave function in region $j(j=\mathrm{I}$, II, III) is

$$
\begin{aligned}
\Psi_{j}= & {\left[a_{j_{1}}\left(\begin{array}{c}
1 \\
\chi_{j_{1}}
\end{array}\right) e^{i k_{\zeta_{1}} \xi}+a_{j_{2}}\left(\begin{array}{c}
1 \\
\chi_{j_{2}}
\end{array}\right) e^{i k_{\xi_{2}} \xi}\right.} \\
& \left.+a_{j_{3}}\left(\begin{array}{c}
1 \\
\chi_{j_{3}}
\end{array}\right) e^{i k_{\xi_{3}} \xi}+a_{j_{4}}\left(\begin{array}{c}
1 \\
\chi_{j_{4}}
\end{array}\right) e^{i k_{\xi_{j_{4}}} \xi}\right] e^{i k_{\eta} \eta},
\end{aligned}
$$

where $k_{\zeta_{n}}(n=1,2,3,4)$ are the four solutions of Eq. (4). In region $\mathrm{I}(\zeta<0)$, an incident mode $k_{\zeta}^{i}=k_{\zeta_{1}}$ propagating to the right with $v_{\zeta}=\left(\partial E / \partial k_{\zeta}\right)_{k_{\eta}}>0$ may be scattered into a reflected mode $k_{\zeta}^{f}=k_{\zeta_{2}}$ propagating towards the left with $v_{\zeta}<0$ and an evanescent mode with $\operatorname{Im}\left(k_{\zeta_{3}}\right)<0$. In region II $(0 \leqslant \zeta \leqslant d)$, four modes exist due to the ultrathin barrier. In region III $(\zeta>d)$, there is a transmitted mode propagating to the right and an evanescent mode with $\operatorname{Im}\left(k_{\zeta_{4}}\right)>0$. Based on the above analysis, we have $a_{\mathrm{I}_{4}}=a_{\mathrm{III}_{2}}=a_{\mathrm{III}_{3}}=0$. We also set $a_{\mathrm{I}_{1}}=1$ in region I to simplify the calculations. The rest eight unknown coefficients can be determined by applying the boundary conditions of the wave functions and probability current, which are given by

$$
\begin{aligned}
\left.\Psi_{\mathrm{I}}\right|_{\zeta=0} & =\left.\Psi_{\mathrm{II}}\right|_{\zeta=0},\left.\quad \Psi_{\mathrm{II}}\right|_{\zeta=d}=\left.\Psi_{\mathrm{III}}\right|_{\zeta=d}, \\
\left.\hat{v}_{\zeta} \Psi_{\mathrm{I}}\right|_{\zeta=0} & =\left.\hat{v}_{\zeta} \Psi_{\mathrm{II}}\right|_{\zeta=0},\left.\quad \hat{v}_{\zeta} \Psi_{\mathrm{II}}\right|_{\zeta=d}=\left.\hat{v}_{\zeta} \Psi_{\mathrm{III}}\right|_{\zeta=d},
\end{aligned}
$$

where $\hat{v}_{\zeta}=\partial \hat{H} / \partial k_{\zeta}$ is the current operator. Then, the unknown coefficients such as the reflection amplitudes $r=a_{\mathrm{I}_{2}}$ can be determined by using the transfer matrix method [43, 44] combined with the boundary conditions in Eq. (6). For $\alpha=0$, Eq. (4) is a quadratic equation about $k_{x}$. There are only two real solutions for $k_{x}$, which means there is no evanescent mode in Eq. (5). Similarly, we can set $a_{\mathrm{I}_{1}}=1$, and the rest four nonzero coefficients can be solved using only the boundary conditions of the wave functions, i.e., $\left.\Psi_{\mathrm{I}}\right|_{x=0}=\left.\Psi_{\mathrm{III}}\right|_{x=0}$, and $\left.\Psi_{\mathrm{III}}\right|_{x=d}=\left.\Psi_{\text {III }}\right|_{x=d}$.

In region $\mathrm{I}$, the interference between the incident and reflected waves gives an oscillation pattern of the LDOS in real space, i.e., the Friedel oscillations [33]. The LDOS near a line defect is [39-42]

$$
\begin{aligned}
\rho(\zeta, E) & =\sum_{\mathbf{k}}\left|\Psi_{1}(\zeta, \eta)\right|^{2} \delta\left(E-E_{\mathbf{k}}\right) \\
& =\int_{1}\left|\Psi_{1}(\zeta, \eta)\right|^{2} \delta\left(E-E_{\mathbf{k}}\right) d \mathbf{k} \\
& =\oint_{E}\left|\Psi_{1}(\zeta, \eta)\right|^{2} d k_{\eta}=\rho_{0}(E)+\delta \rho(\zeta, E),
\end{aligned}
$$

where $\rho_{0}(E)$ is spatially independent, and it can be ignored. In real scanning tunneling microscope experiments [34-38], one often measures the spatially dependent part $\delta \rho(\zeta, E)$, which is given by

$$
\delta \rho(\zeta, E)=\oint_{E} \operatorname{Re}\left[r\left(1+\chi_{\mathrm{I}_{1}}^{*} \chi_{\mathrm{I}_{2}}\right) e^{i\left(k_{\zeta}^{f}-k_{\zeta}^{i}\right) \zeta}\right] d k_{\eta}+\delta \rho_{1}(\zeta, E),
$$

where $\delta \rho_{1}(\zeta, E)$ originates from the evanescent mode and decays to zero quickly for positions far away from the line defect. Therefore, the LDOS oscillation is dominated by the first term in Eq. (8). For positions away from the defect, the LDOS oscillation sensitively depends on the phase factor $e^{i\left(k_{\zeta}^{f}-k_{\zeta}^{i}\right) \zeta}$ which oscillates rapidly. A pair of scattering sates $\left(k_{\zeta}^{i}, k_{\eta}\right)$ and $\left(k_{\zeta}^{f}, k_{\eta}\right)$ on the CEC result in a standing wave with spatial period of $2 \pi /\left|\left(k_{\zeta}^{f}-k_{\zeta}^{i}\right)\right| \equiv 2 \pi /\left|\Delta k_{\zeta}\right|$. Only the pair whose period is stationary with respect to small variation in $k_{\eta}$ makes dominant contribution to the LDOS oscillations [39, 41, 42]. Those pairs of points on the CEC are called as stationary phase points, which satisfy

$$
\left.\frac{\partial \Delta k_{\zeta}}{\partial k_{\eta}}\right|_{k_{\eta 0}}=\left.\frac{\partial\left(k_{\zeta}^{f}-k_{\zeta}^{i}\right)}{\partial k_{\eta}}\right|_{k_{\eta 0}}=0 .
$$

The stationary phase points given by Eq. (9) can be divided into two categories according to the sign of the second derivative $\left(\partial^{2} \Delta k_{\zeta} / \partial k_{\eta}^{2}\right)$ in the neighbourhood of these points. One category is the extreme points (EPs) around which the second derivatives have the same signs [39, 41, 42]. In this case, the wavevector changes $\Delta k_{\zeta}$ are maximum or minimum values on the CEC. Another category is the inflection points around which the second derivatives have opposite signs. In our work, we only encounter the EPs. Between a pair of EPs, $\Delta k_{\zeta 0}=k_{\zeta 0}^{f}-k_{\zeta 0}^{i}$ is the characteristic wavevector solely determined by the geometry of CEC. The spatial dependence of the LDOS can be evaluated by expanding the relevant quantities in Eq. (8) to the lowest leading order about $\delta k_{\eta}$ around each pair of EPs, which is given by

$$
\begin{aligned}
k_{\eta} & \rightarrow k_{\eta 0}+\delta k_{\eta}, \quad r \rightarrow r_{0} \delta k_{\eta}^{\beta_{\zeta}}, \\
1+\chi_{\mathrm{I}_{1}}^{*} \chi_{\mathrm{I}_{2}} & \rightarrow c_{0} \delta k_{\eta}^{\gamma_{\zeta}}, \quad k_{\zeta}^{f}-k_{\zeta}^{i} \rightarrow \Delta k_{\zeta 0}+\Delta k_{\zeta \lambda} \delta k_{\zeta}^{\lambda_{\zeta}},
\end{aligned}
$$

Then, the asymptotic behavior of the LDOS is

$$
\delta \rho(\zeta, E) \simeq \rho_{A} \cos \left(\Delta k_{\zeta 0} \zeta+\phi\right) \zeta^{\nu},
$$

where $\rho_{A}=\operatorname{Re}\left(i^{-v} c_{0} r_{0} / \lambda_{\zeta}\right) \Gamma(-v)\left(\Delta k_{\zeta \lambda}\right)^{v}$ is the amplitude of LDOS pattern, $\Delta k_{\zeta \lambda}$ is the lowest leading order derivative of $\Delta k_{\zeta}\left(k_{\eta}\right)$ at $\left(k_{\xi}^{i}, k_{\eta}\right), v=-\left(\beta_{\zeta}+\gamma_{\zeta}+1\right) / \lambda_{\zeta}$ is the power-law decay index, $\Gamma(x)$ is the Euler function, and $\phi$ is the initial phase of each pair of EPs. The asymptotic decay behavior of LDOS oscillations in Eq. (11) is valid if $\zeta \gg\left|\Delta k_{\zeta 0}\right|^{-1}[39,41,42]$, which means the asymptotic region is energy dependent.

\section{LOCAL DENSITY OF STATES OSCILLATIONS}

In this section, we present some numerical examples of the LDOS oscillations when the line defects are along different directions for the system with and without a gap, respectively. In order to understand the numerical results better, we analytically obtain the power-law decay indexes of the LDOS oscillations for two special cases within the stationary phase approximation.

First, we consider the LDOS oscillation of $\alpha=0$ for the gapless case $(\Delta=0)$. Fig. 2(a) plots the reflectivity 

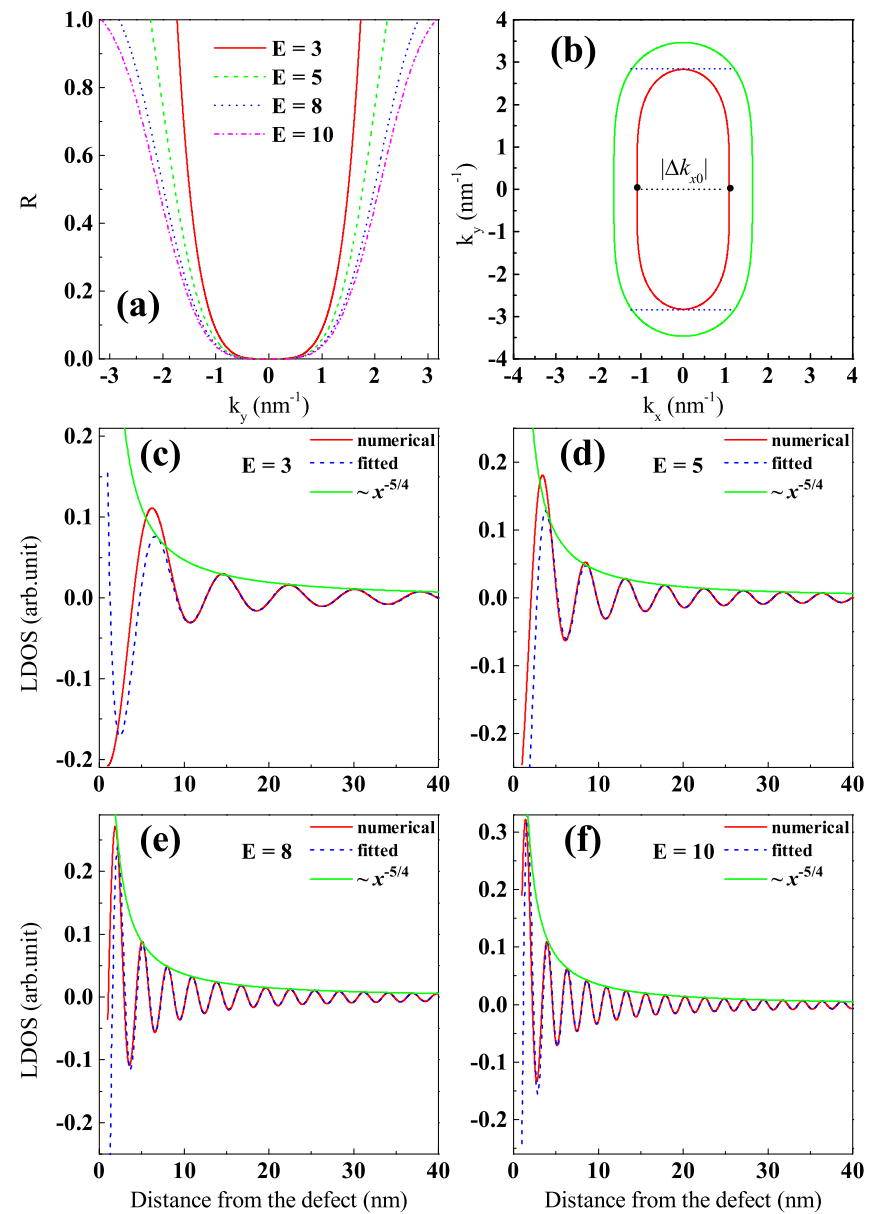

FIG. 2: (a) The reflectivity $R$ as a function of $k_{y}$ at different energies for $\alpha=0$. (b) The CECs of the incident (red line) and scattering (green line) regions with $E=8$. The black solid dots are a pair of stationary phase points, and $\left|\Delta k_{x 0}\right|$ is the characteristic wave vector of the scattering process. (c)-(f) The spatial dependence of LDOS at different energies. The red solid (blue dashed) lines are the numerical (fitted) results. The green solid lines are the envelop function of LDOS. In all figures, we have set $U_{0}=20$ and $d=1$.

$R=|r|^{2}$ as a function of the transverse wave vector $k_{y}$ with different energies. As shown in the figure, the reflectivity $R$ is always zero at normal incidence i.e., $k_{y}=0$, due to the Klein tunneling resulting from the time-reversal symmetry of the effective Hamiltonian [30, 45]. It increases at oblique incidence with increasing incident angle due to the mismatch of the wave vector [30,31]. Fig. 2(b) depicts the CECs of the incident area (red line) and the barrier area (green line). As plotted in the figure, the scattering only occurs in the range within the blue dashed lines resulting from the conservation of $k_{y}$. Figs. 2(c-f) present the spatial dependence of LDOS with $E=3,5,8,10$, respectively. As depicted in the figure, numerical results (the solid red lines) indicate that the LDOS periodically oscillates with the distance away from the defect with a decreasing amplitude, which implies an asymptotic behavior.

As discussed in Sec II, the LDOS oscillation can be understood by using the stationary phase approximation. First, by using Eq. (9), the pair of stationary phase points on the CEC are $(E / u, 0)$ and $(-E / u, 0)$ [see the black solid dots in Fig. 2(b)]. The second derivative $\left(\partial^{2} \Delta k_{x} / \partial k_{y}^{2}\right)$ at these points are zero but negative in the neighbourhood of them. Hence, these two points $[( \pm E / u, 0)]$ constitute a pair of maximum points, giving the characteristic wave vector as $\left|\Delta k_{x 0}\right|=|-2 E / u|$. Therefore, the period of the LDOS oscillation is $2 \pi /\left|\Delta k_{x 0}\right|=\pi u / E$, which means the higher the incident energy, the faster the LDOS oscillations. This explains why the LDOS oscillates faster for higher energy in Figs. 2(c-f). In order to get the power-law decay index, we need to expand relevant quantities around the EPs. Because the first, second, and third derivatives of $\Delta k_{x}\left(k_{y}\right)$ at the EPs are all zero, we have to expand it to the fourth-order. Then, near the EPs, we have $\Delta k_{x} \approx \Delta k_{x 0}+\delta k_{y}^{4} / u E, 1+\chi_{\mathrm{I}_{1}}^{*} \chi_{\mathrm{I}_{2}} \approx-2 i \delta k_{y}^{2} / E$, and $\chi_{\mathrm{I}_{1}}-\chi_{\mathrm{II}_{1}} \approx\left[1 / E-1 /\left(E-U_{0}\right)\right] \delta k_{y}^{2}$. Therefore, the relevant parameters are $\lambda_{x}=4, \gamma_{x}=2$ and $c_{x 0}=-2 i / E$. To obtain the parameter $\beta_{x}$, we need to calculate the reflectivity amplitude. For a given $E$ and $k_{y}$, using the continuity condition of the wave function, the reflectivity amplitude is obtained as

$$
r=-i \frac{2\left(\chi_{\mathrm{I}_{1}}-\chi_{\mathrm{II}_{1}}\right)\left(\chi_{\mathrm{I}_{1}}-\chi_{\mathrm{II}_{1}}^{*}\right) \sin \left(q_{x} d\right)}{\left|\chi_{\mathrm{I}_{1}}-\chi_{\mathrm{II}_{1}}\right|^{2} e^{i q_{x} d}-\chi_{\mathrm{I}_{1}}-\left.\chi_{\mathrm{II}_{1}}^{*}\right|^{2} e^{-i q_{x} d}},
$$

where $k_{x}=\sqrt{E^{2}-k_{y}^{4}} / u$ and $q_{x}=\sqrt{\left(U_{0}-E\right)^{2}-k_{y}^{4}} / u$. Expanding the reflectivity amplitude near the EPs, we have $r \approx r_{x 0} \delta k_{y}^{2}$ with $r_{x 0}=\sin \left(U_{0} d / u\right) e^{-i U_{0} d / u} E^{-1}$, giving $\beta_{x}=$ 2. One can also obtain the parameter $\beta_{x}$ by fitting the reflectivity amplitude as a polynomial of $\delta k_{y}$ numerically, which is easier than calculating the reflectively amplitude analytically. Hereafter, we will use numerical fitting to obtain this parameter. Then, the power-law decay index is $v=$ $-\left(\beta_{x}+\gamma_{x}+1\right) / \lambda_{x}=-5 / 4$. Therefore, according to the analysis, the LDOS oscillation in this case can be fitted as $\delta \rho(x, E) \simeq \rho_{A} \cos \left(-\frac{2 E}{u} x+\phi\right) x^{-\frac{5}{4}}$. Based on Eq. (11), the amplitude $\rho_{A}$ depends on $r_{x 0}, c_{x 0}$ and the forth derivative of $\Delta k_{x}\left(k_{y}\right)$ at $(E / u, 0)$. Taking all the factors together, we find the amplitude is proportional to $E^{-3 / 4}$ which means the higher the incident energy, the smaller the amplitude. Unfortunately, we cannot directly observe it in Fig. 2 because different incident energy corresponds to different pair of EPs having different initial phase. The blue dashed lines in Figs. 2(c-f) show that the fitted results for the LDOS are in excellent agreement with the numerical ones. The asymptotic lines $x^{-5 / 4}$ well describe the asymptotic behavior of the LDOS, which clearly demonstrate that the decay index is $-5 / 4$. However, the LDOS oscillations close to the line defect departure from the asymptotic behavior because the asymptotic region requires $x \gg\left|\Delta k_{x 0}\right|^{-1}=u / 2 E[39,41,42]$. This means that the higher the incident energy, the smaller the distance needed to manifest asymptotic behavior. We can also directly observe this feature in Figs. 2(c-f). More precisely, based on our calculation, we find the asymptotic behavior expressed by Eq. (11) works quite well if $x$ is one order larger than $\left(\Delta k_{x 0}\right)^{-1}$.

On the other hand, it is worth to point out that the decay index $-5 / 4$ here is not only different from the decay of $-3 / 2$ for isotropic Dirac materials [38-41] but also the decay 

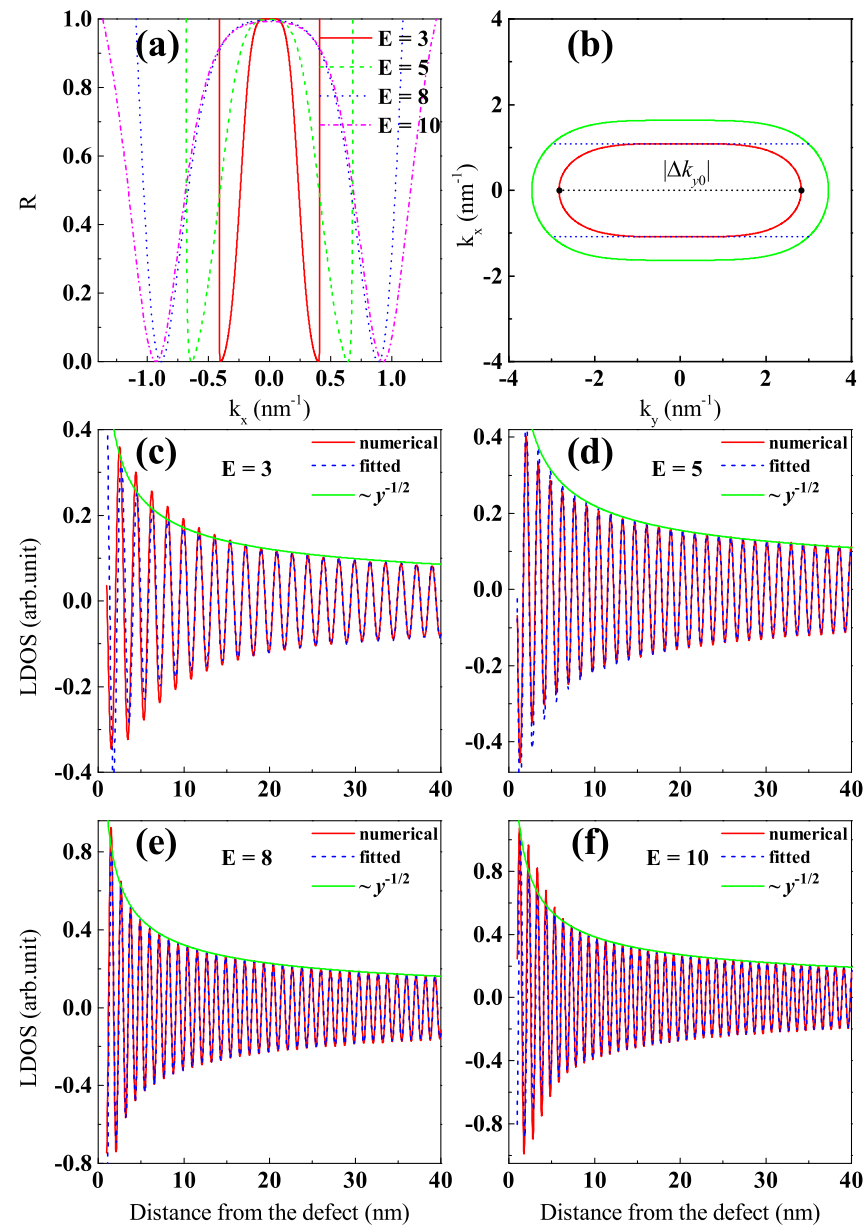

FIG. 3: (a) The reflectivity $R$ as a function of $k_{x}$ at different energies for $\alpha=\pi / 2$. (b) The CECs of the incident (red line) and scattering (green line) regions with $E=8$. The black solid dots are a pair of stationary phase points, and $\left|\Delta k_{y 0}\right|$ is the characteristic wave vector during the scattering process. (c)-(f) The LDOS oscillations at different energies. The red solid (blue dashed) lines are the numerical (fitted) results. The green solid lines are the envelop function of LDOS. Other parameters are the same as those in figure 2.

of $-1 / 2$ for conventional semimetals $[36,37,41]$. This unique decay index originates from the unique anisotropic band structure around SDP. In particular, compared with conventional semimetals, there is Klein tunneling suppressing the backscattering at normal incidence, promising a faster decay than that of $-1 / 2$ [39]. In contrast to isotropic Dirac systems, electrons are more difficult to transmit the barrier due to the severer mismatch of the wave vector at oblique incidence, resulting in a slower decay behavior than that of $-3 / 2$. Noteworthily, from the derivation, the decay index $-5 / 4$ is independent on the incident energy, the barrier height or the band parameters. Therefore, the decay index $-5 / 4$ can serve as a fingerprint to characterize 2D semi-Dirac electrons.

Next, we turn to another special case of $\alpha=\pi / 2$. Fig. 3(a) shows the reflectivity $R$ as a function of the transverse wave vector $k_{x}$ with various energies. In contrast to the case of $\alpha=$ 0 , the reflectivity here is no longer zero at normal incidence i.e., $k_{x}=0$, due to the absence of Klein tunneling. Instead, there is a total reflection at normal incidence resulting from the server mismatch of the wave vectors [see Eq. (4)] between the incident and scattering states because of the high electrical barrier. This is consistent with the previous results [30]. Similarly, Figs. 3(c-f) depict the LDOS oscillations in this case with $E=3,5,8,10$, respectively. As shown in the figure, numerical results (the solid red lines) indicate that the LDOS periodically oscillates with distance away from the defect with decreasing amplitude, which also implies an asymptotic behavior in the oscillation pattern. Fig. 3(b) plots the CEC of the incident and barrier regions, respectively. From Fig. 3(b), we find a pair of EPs $(0, \sqrt{E})$ and $(0,-\sqrt{E})$ satisfying Eq. (9) (see the black solid dots), giving the characteristic wave vector as $\left|\Delta k_{y 0}\right|=2 \sqrt{E}$. Hence, the period of the LDOS oscillation in this case is $2 \pi /\left|\Delta k_{y 0}\right|=\pi / \sqrt{E}$, which also means the higher the energy, the faster the oscillation pattern. Those features are well reflected in Figs. 3(c)-(f). Meanwhile, for a certain incident energy, the period here is smaller than that in the case of $\alpha=0$. Following the same process of $\alpha=0$, we can also obtain the asymptotic behavior of the LDOS by using the stationary phase approximation. Specifically, near the EPs, we have $\Delta k_{y} \approx \Delta k_{y 0}+u^{2} \delta k_{x}^{2} /(2 E \sqrt{E}), 1+\chi_{\mathrm{I}_{1}}^{*} \chi_{\mathrm{I}_{2}} \approx 2$. Hence, the relevant parameters are $\lambda_{y}=2, \gamma_{y}=0$, and $c_{y 0}=2$. As plotted in Fig. 3(a), the reflectivity is almost unit around the $\mathrm{EP}$, giving the parameter $\beta_{y}=0$ and $r_{y 0}=1$. Therefore, the power-law decay index in this case is $v=-\left(\beta_{y}+\gamma_{y}+1\right) / \lambda_{y}=$ $-1 / 2$, which means the spatial dependence of the LDOS can be expressed as $\delta \rho(y, E) \simeq \rho_{A} \cos (-2 \sqrt{E} y+\phi) y^{-\frac{1}{2}}$ with $\rho_{A}=\pi\left(\partial^{2} \Delta k_{y} / \partial k_{x}^{2}\right)^{-1 / 2} / \sqrt{2}$. In this case, the amplitude $\rho_{A}$ only depends on the reciprocal of the square root of the second derivative $\left(\partial^{2} \Delta k_{y} / \partial k_{x}^{2}=u^{2} / E^{3 / 2}\right)$, which is just the square root of the curvature at EPs on the CEC. The blue dashed lines in Figs. 3(c-f) show the results fitted with the above formula for the LDOS. As expected, the fitted results are in good agreement with the numerical ones. And, the asymptotic line $y^{-1 / 2}$ well describes the asymptotic behavior of the LDOS far away from the line defect. This decay index is the same as that in conventional metals $[36,37,41]$ because both of them have a parabolic dispersion. In the very vicinity of the line defect, the LDOS oscillations departure from the asymptotic behavior because the asymptotic region requires $y \gg\left|\Delta k_{y 0}\right|^{-1}=1 / 2 \sqrt{E}[39,41,42]$. This means that the higher the incident energy, the smaller the distance needed to manifest asymptotic behavior. We can also directly observe this feature in Figs. 3(c-f). Specifically, we find that the asymptotic behavior starts when $y$ is twenty times larger than $\left(\Delta k_{y 0}\right)^{-1}$ based on our calculation.

Next, we discuss the case of $0<\alpha<\pi / 2$, which means that the line defect is along an arbitrary direction. In contrast to the two special cases, the decay indexes in this case sensitively depend on the incident energy and the orientation of the defect i.e., the titled angle $\alpha$, because the CECs are titled ellipses. Here, we choose the results for $\alpha=\pi / 4$ as an example to illustrate this feature. Figs. 4(a)-(b) show 

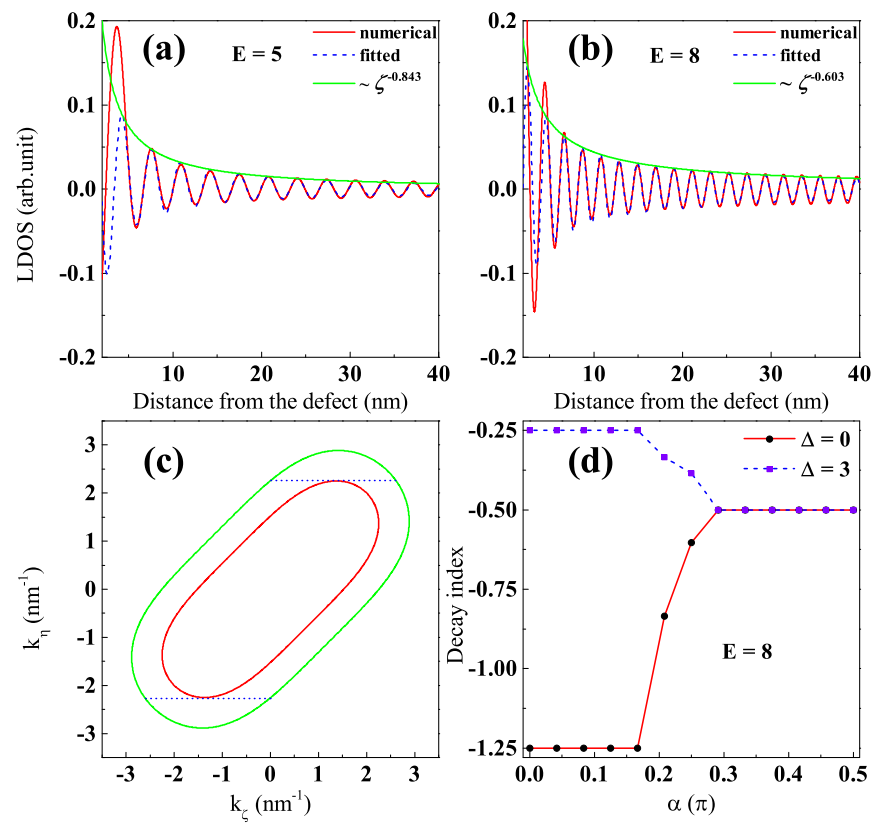

FIG. 4: (a)-(b) The LDOS oscillations at different energies for $\alpha=\pi / 4$. (c) The CECs of the incident (red line) and scattering (green line) regions with $E=8$. (d) The power-law decay index as a function the titled angle $\alpha$ with incident energy $E=8$. The red solid (blue dashed) line is the result for the system without (with) a gap. Other parameters are the same as those in figure 2.

the LDOS oscillations near the defect for $E=5$ and 8, respectively. From the figures, we find that the numerical results (the red solid lines) also imply an asymptotic behavior in the oscillation pattern. Following the analysis for the two special cases ( $\alpha=0$ and $\pi / 2$ ), we can also try to fit the LDOS by using a similar formula like Eq. (11). The fitted results and asymptotic lines are indicated by the blue dashed and green solid lines in the figures. The decay indexes here are found to be -0.843 and -0.603 for $E=5$ and 8 , respectively. We have also checked various cases with different incident energies and $\alpha$, but do not present them here due to space limitations. The LDOS oscillations for other angle $\alpha$ and incident energies are similar to the results shown in Figs. 4(a)-(b). However, the decay indexes are distinct from each other and sensitively depend on the incident energy and $\alpha$. The reason is that the CECs are titled ellipses as depicted in Fig. 4(c) and there is no such a strict stationary phase point on the CEC satisfying Eq. (9). The red solid line in Fig. 4(d) plots the decay indexes for various titled angle $\alpha$ with incident energy $E=8$ by fitting with the numerical results. The result shows that the decay index sensitively depends on the orientation of the defect $(\alpha)$ and varies from $-5 / 4$ to $-1 / 2$.

To explore whether the decay index $-5 / 4$ is unique, we assume a gap $(\Delta)$ in Hamiltonian (1) and redo the calculation. Figs. 5(a)-(b) show the reflectivity as a function of transverse wave vectors for $\alpha=0$ and $\pi / 2$, respectively. As shown in Fig. 5 (a), for $\alpha=0$, the reflectivity is finite at normal incidence due to the absence of Klein tunneling resulting from the
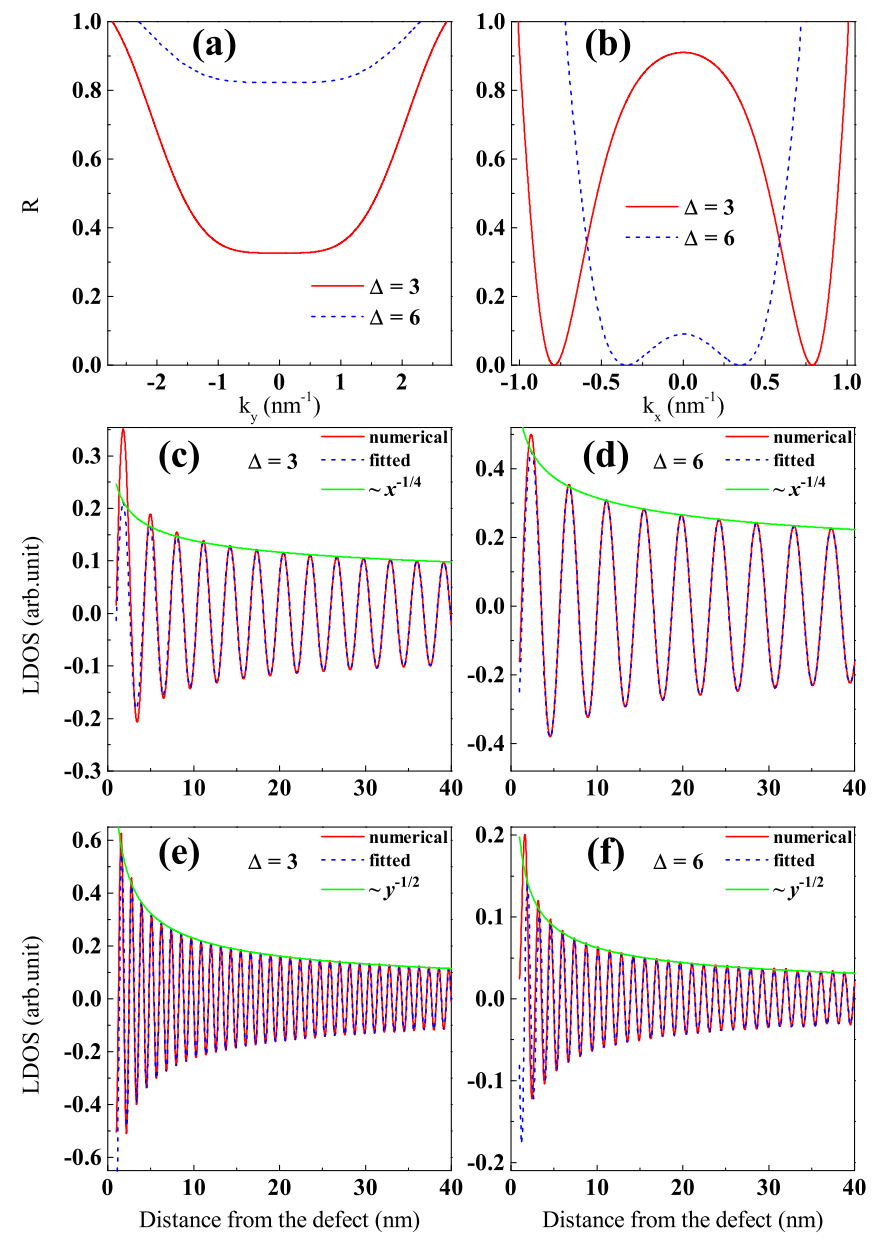

FIG. 5: (a)/(b) Reflectivity as a function of transverse wave vectors for $\alpha=0 / \frac{\pi}{2}$ with different band gaps. (c)-(d) The LDOS oscillations with different band gaps for $\alpha=0$. (e)-(f) The LDOS oscillations with different band gaps for $\alpha=\pi / 2$. The electron energy is $E=8$, and other parameters are the same as those in figure 2.

breaking of time reversal symmetry, which is different from the result of the gapless case. For $\alpha=\pi / 2$, the reflectivity is nonzero at normal incidence regardless of whether there is a band gap. This is similar to that of the gapless case. Figs. 5(c)-(f) plot the LDOS oscillations for $\alpha=0$ and $\pi / 2$ with different band gaps. Similar to the results of the gapless case, the LDOSs oscillate periodically with a decay magnitude near the line defect, which can be fitted by an analytical function as expressed in Eq. (11). Here, for the case of $\alpha=0$, the stationary phase points on the CEC are $\left(k_{x 0}^{\prime}, 0\right)$ and $\left(-k_{x 0}^{\prime}, 0\right)$ with $k_{x 0}^{\prime}=\sqrt{E^{2}-\Delta^{2}} / u$, giving the period of the LDOS oscillations as $\pi u / \sqrt{E^{2}-\Delta^{2}}$. This means the larger the band gap, the slower the oscillation pattern for a certain incident energy. Meanwhile, the asymptotic region falls into $x \gg u / 2 \sqrt{E^{2}-\Delta^{2}}$ which means the larger the band gap, the longer the distance needed to manifest asymptotic behavior for a certain energy. Those features can be clearly observed in Figs. 5(c)-(d). Near those two points, 
we have $\Delta k_{x}^{\prime} \approx-2 k_{x 0}^{\prime}+\delta k_{y}^{4} / u \sqrt{E^{2}-\Delta^{2}}$ and $1+\chi_{\mathrm{I}_{1}}^{*} \chi_{\mathrm{I}_{2}} \approx$ $2 \Delta /(E+\Delta)$. Thus, the relevant parameters are $\lambda_{x}^{\prime}=4$, $\gamma_{x}^{\prime}=0$, and $c_{x 0}^{\prime}=2 \Delta /(E+\Delta)$. The reflectivity amplitude around $k_{y}=0$ is $r \approx r_{x 0}^{\prime}$ with $r_{x 0}^{\prime}=U_{0} \Delta /\left[2 E\left(E-U_{0}+\Delta\right)-U_{0} \Delta+\right.$ $\left.i \sqrt{u q_{x}^{\prime}\left(E^{2}-\Delta^{2}\right)} \cot \left(q_{x}^{\prime} d\right)\right]$ and $q_{x}^{\prime}=\sqrt{\left(U_{0}-E\right)^{2}-\Delta^{2}} / u$, giving the parameter $\beta_{x}^{\prime}=0$ which is different from the result of the gapless case. Therefore, the power-law decay index for gapped semi-Dirac system is $v=-\left(\beta_{x}^{\prime}+\gamma_{x}^{\prime}+1\right) / \lambda_{x}^{\prime}=$ $-1 / 4$. The LDOS oscillations can be fitted as $\delta \rho(x, E) \simeq$ $\rho_{A x}^{\prime} \cos \left(-2 k_{x 0}^{\prime} x+\phi\right) x^{-\frac{1}{4}}$. The amplitude $\rho_{A x}^{\prime}$ depends on $r_{x 0}^{\prime}$, $c_{x 0}^{\prime}$ and the forth derivative of $\Delta k_{x}^{\prime}\left(k_{y}\right)$ at $\left(k_{x 0}^{\prime}, 0\right)$. It is a complex function of the incident energy and band gap. The blue dashed lines in Figs. 5(c-d) show the fitted results for the LDOS. They are in good agreement with the numerical ones. Moreover, the envelop function $x^{-1 / 4}$ perfectly describes the asymptotic behavior of the LDOS. It is worth to point out that the $-1 / 4$ decay behavior of the LDOS is also valid near the bottom (top) of the conduction (valence) band for small band gaps. For higher energy LDOS oscillations with small gap, the decay index may be different, but it is usually difficult to realize in real experiments because higher Fermi surface requires high carrier density in the sample. On all accounts, the decay index can't be $-5 / 4$ (the decay index of gapless case) as long as the SDP is gapped, which further indicates that the $-5 / 4$ decay behavior can serve as a fingerprint to verify the SDP in $2 \mathrm{D}$ electron system. Similarly, for the case of $\alpha=\pi / 2$, the extreme points are $\left(0, k_{y 0}^{\prime}\right)$ and $\left(0,-k_{y 0}^{\prime}\right)$ with $k_{y 0}^{\prime}=\left(E^{2}-\Delta^{2}\right)^{1 / 4}$. Near those two points, we have $\Delta k_{y}^{\prime} \approx-2 k_{y 0}^{\prime}+u^{2} \delta k_{x}^{2} / 2\left(E^{2}-\Delta^{2}\right)^{3 / 4}$, and $1+\chi_{\mathrm{I}_{1}}^{*} \chi_{\mathrm{I}_{2}} \approx 2 E /(E+\Delta)$, which results in the relevant parameters $\lambda_{y}^{\prime}=2$ and $\gamma_{y}^{\prime}=0$. It can be seen from Fig. 5(b) that the reflection amplitude is not zero near the extreme points, thus, we obtain $\beta_{y}^{\prime}=0$. Hence, the decay index in this case is $v=-\left(\beta_{y}^{\prime}+\gamma_{y}^{\prime}+1\right) / \lambda_{y}^{\prime}=-1 / 2$, which is same as that of the gapless case. Therefore, the LDOS oscillation can be fitted as $\delta \rho(y) \propto \cos \left(-2 k_{y 0}^{\prime} y+\phi\right) y^{-\frac{1}{2}}$. The amplitude of the LDOS in this case is a complex function of the incident energy and band gap. It is more easily to obtain it by fitting with the numerical data. The blue dashed lines in Figs. 5(e)-(f) are the fitted results, which are in good agreement with the numerical results. The envelop function $y^{-1 / 2}$ perfectly governs the asymptotical behavior of the LDOS oscillations, which indicates that the powerlaw decay index is $-1 / 2$ for defects perpendicular to the parabolic dispersion direction regardless of whether the SDP is gapped or not. The asymptotic region in this case is $x \gg$ $\left(E^{2}-\Delta^{2}\right)^{-1 / 4} / 2$ which also indicates that the larger the band gap, the longer the distance needed to manifest asymptotic behavior for certain energies. For $0<\alpha<\pi / 2$, the decay indexes also depend on the incident energy and the orientation of the defect. The blue dashed line in Fig. 4(d) plots the decay indexes for various titled angle $\alpha$ with incident energy $E=8$ and gap $\Delta=3$ by fitting with the numerical results. From the figure, we find that the decay index depends on the orientation of the defect and varies from $-1 / 4$ to $-1 / 2$.

\section{SUMMARY}

In summary, using quantum mechanical scattering theory and the method of stationary phase approximation, we studied the LDOS oscillations near a line defect in the semi-Dirac electron system and analytically obtained the power-law decay indexes for two special orientations of the defect. When the line defect is perpendicular to the linear dispersion direction, the decay index is $-5 / 4$ for gapless SDP and $-1 / 4$ when the SDP is gapped, both of them are different from the decay index $-3 / 2$ in isotropic Dirac systems. When the line defect is perpendicular to the parabolic dispersion direction, the decay index is always $-1 / 2$ regardless of whether the SDP is gapped or not. This is the same as that of conventional metals because both of them have a parabolic dispersion. There is no such a universal decay index when the line defect runs along an arbitrary direction due to the absence of stationary phase points on the CEC. Our results can be tested by the scanning tunnelling microscope [34-38], and the decay index $-5 / 4$ provides a fingerprint to detect semiDirac electrons. The power-law decay behavior is more likely to manifest itself at higher Fermi levels, which means it is more easily to be observed in samples with higher carrier concentrations.

\section{Acknowledgments}

This work was supported by the National Natural Science Foundation of China (Grant Nos. 11804092 and 11774085), Project funded by China Postdoctoral Science Foundation (Grant Nos. BX20180097, 2019M652777), and Hunan Provincial Natural Science Foundation of China (Grant No. 2019JJ40187).

\section{Appendix}

In this appendix, we discuss the LDOS oscillations near the line defect which is modeled as a $\delta$-potential. When the line defects run along the linear dispersion direction, the potential profile is $U(x)=U_{0} \delta(x)$, which is the limitation of the rectangular potential $U(x)=U_{0} \Theta(x) \Theta(d-x)$ if $U_{0} \rightarrow \infty, d \rightarrow 0$, and $U_{0} d \equiv$ constant. Since the momentum is conserved in the $y$-direction, we can express the wavefunction as $\Psi_{\mathbf{k}}(x, y)=$ $e^{i k_{y} y} \varphi(x)$. Because the secular equation $\left[H\left(-i \partial_{x}, k_{y}\right)+\right.$ $\left.U_{0} \delta(x)\right] \Psi_{\mathbf{k}}(x, y)=E \Psi_{\mathbf{k}}(x, y)$ is a first-order partial differential equation with respect to $x$, the wavefunction is discontinuous at $x=0$. The boundary condition at $x=0$ is given by [46, 47]

$$
\left.\Psi_{\mathrm{k}}\right|_{x=0^{+}}=\left.e^{-i \sigma_{y} \tau} \Psi_{\mathrm{k}}\right|_{x=0^{-}},
$$

where $\tau=U_{0} d / u$ is a constant. By using Eq. (A1), we obtain the reflection amplitude as

$$
r=\frac{\left(k_{y}^{4}+i u k_{x} k_{y}^{2}\right) \sin \tau}{i u k_{x} E \cos \tau-E^{2} \sin \tau} .
$$

Following the procedure of stationary phase approximation, 

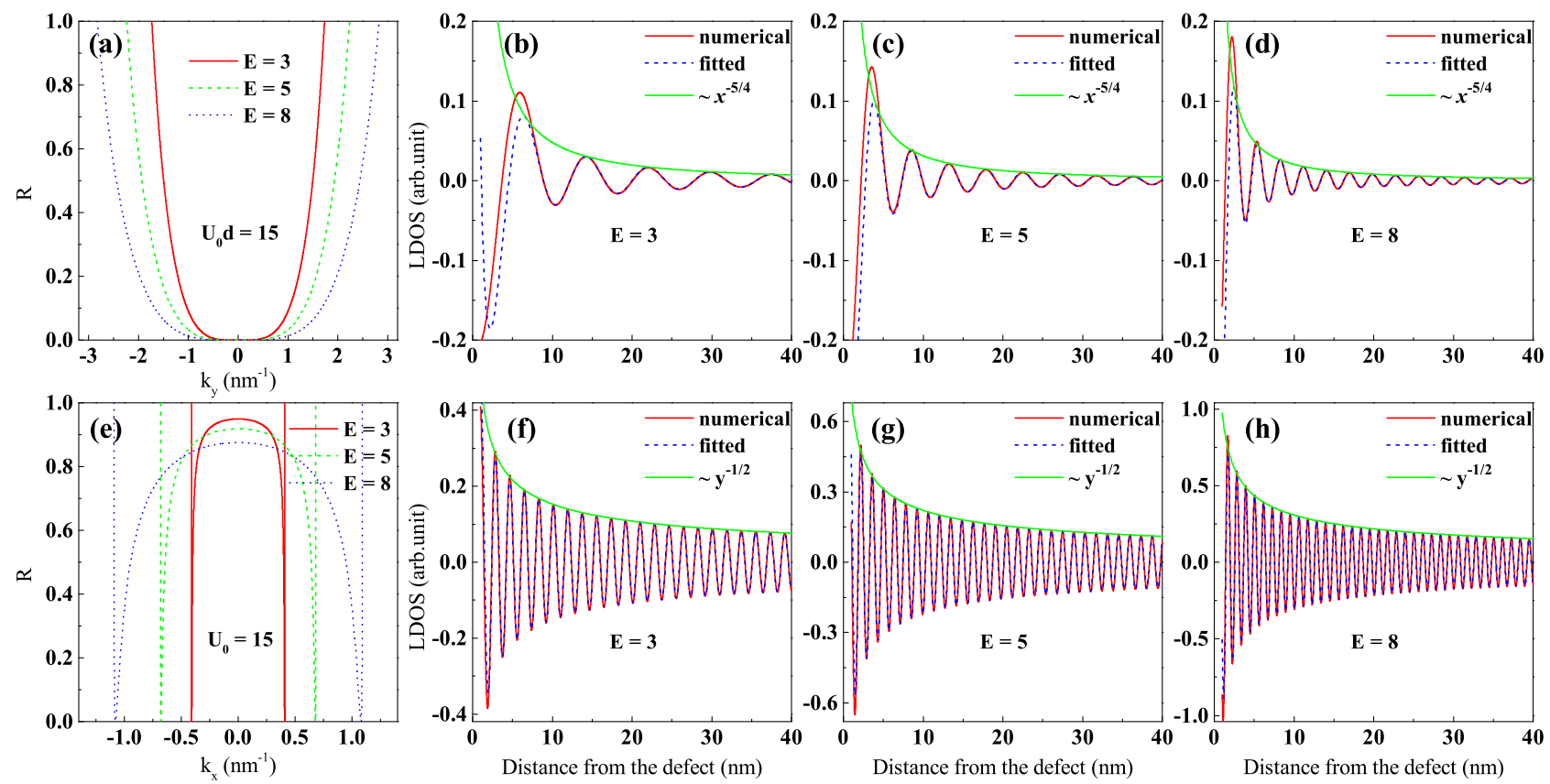

FIG 6: The first (second) row is the result for the defect perpendicular to the linear (parabolic) dispersion direction. (a)/(e) The reflectivity $R$ as a function of $k_{y} / k_{x}$ at different energies. (b)-(d) and (f)-(h) The spatial dependence of LDOS at different energies. The red solid (blue dashed) lines are the numerical (fitted) results. The green solid lines are the envelop function of LDOS. The strength of the $\delta$ potential is $U_{0} d=15$ $\left(U_{0}=15\right)$ when the line defect is perpendicular to the linear (parabolic) dispersion direction.

expanding the reflectivity amplitude near the EPs $( \pm E / u, 0)$ gives $r=\delta k_{y}^{2} \sin \tau /\left(E e^{i \tau}\right)$ which means the parameter $\beta_{x}=2$ which is the same as that in the case of rectangular potential. The other two relevant parameters $\lambda_{x}$ and $\gamma_{x}$ are solely determined by the CEC and independent on the choice of potential, i.e., $\lambda_{x}=4, \gamma_{x}=2$. Therefore, the power-law decay index here remains $v=-\left(\beta_{x}+\gamma_{x}+1\right) / \lambda_{x}=-5 / 4$ which is the same as that in the main text. Figs. $6(\mathrm{a})$ and $6(\mathrm{~b}-\mathrm{d})$ plot the reflectivity $R=|r|^{2}$ and LDOS oscillations counterpart to Figs. 2(a) and 2(c-e). As shown in the figures, we find there is only a little bit quantitative difference between the reflectivity and LDOS oscillations caused by the $\delta$ potential and the rectangular one. The numerical (the red solid lines) and fitted (the blue dashed lines) results both clearly demonstrate that the power-law decay index of LDOS oscillations are identical, i.e., decaying as $x^{-5 / 4}$.

When the line defect runs along the parabolic dispersion direction, the potential profile is $U(y)=U_{0} \delta(y)$. In this case, the wave function admits the form $\Psi_{\mathbf{k}}(x, y)=$ $e^{i k_{x} x} \varphi(y)$. Because the Schrödinger equation $\left[H\left(-i \partial_{y}, k_{x}\right)+\right.$ $\left.U_{0} \delta(y)\right] \Psi_{\mathbf{k}}(x, y)=E \Psi_{\mathbf{k}}(x, y)$ is a second-order partial differential equation with respect to $y$, the boundary condition is [48]

$$
\begin{gathered}
\left.\Psi_{\mathrm{k}}\right|_{y=0^{-}}=\left.\Psi_{\mathrm{k}}\right|_{y=0^{+}}, \\
\sigma_{x}\left(\left.\partial_{y} \Psi_{\mathrm{k}}\right|_{y=0^{+}}-\left.\partial_{y} \Psi_{\mathrm{k}}\right|_{y=0^{-}}\right)=\left.U_{0} \Psi_{\mathrm{k}}\right|_{y=0} .
\end{gathered}
$$

According to Eq. (A3), the reflection amplitude is obtained as

$$
r=\frac{U_{0}^{2} k_{y}-2 U_{0} E}{2 i\left(U_{0} E-2 k_{y}^{3}\right)-\left(U_{0}^{2} k_{y}-2 U_{0} E\right)} .
$$

Similarly, expanding the reflectivity amplitude near the pair of extreme points $(0, \pm \sqrt{E})$ gives $r=U_{0} /\left(2 i \sqrt{E}-U_{0}\right)$ which means the parameter $\beta_{y}=0$. It is the same as that in the case of rectangular potential. The other two relevant parameters $\lambda_{y}$ and $\gamma_{y}$ are solely determined by the CEC and independent on the choice of potential profile. Therefore, the power-law decay index here remains $-1 / 2$ which is identical to that in the case of rectangular potential. Figs. 6(e) and 6(f-h) plot the reflectivity $R=|r|^{2}$ and LDOS oscillations counterpart to Figs. 3 (a) and $3(\mathrm{c}-\mathrm{e})$. From the figures, we find there is only a little bit quantitative difference between the reflectivity and LDOS oscillations caused by the $\delta$ potential and the rectangular one. The numerical (the red solid lines) and fitted (the blue dashed lines) results both clearly demonstrate that the power-law decay index of LDOS oscillations are identical, i.e., decaying as $y^{-1 / 2}$.

In summary, different choices of the potentials will not bring about different decay indexes. The reason is that the quasiparticle interference pattern only depends on the energy dispersion, i.e., the CEC of the system. The scattering potentials can not change the geometry of the Fermi surface or the stationary phase points. Hence, the power-law decay indexes of the LDOS oscillations caused by the line defect are independent on whether choose a $\delta$-potential or a rectangular one to model it. 
[1] K. S. Novoselov, A. K. Geim, S. V. Morozv, D. Jiang, Y. Zhang, S. V. Dubonos, I. V. Grigorieva, and A. A. Firsov, Science 306, 666 (2004).

[2] A. H. C. Neto, F. Guinea, N. M. R. Peres, K. S. Novoselov, and A. K. Geim, Rev. Mod. Phys. 81, 109 (2009).

[3] M. I. Katnelson, K. S. Novoselov and A. K. Geim, Nat. Phys. 2, 620 (2006).

[4] Y. Zhang, Y.-W. Tan, H. L. Stormer and P. Kim, Nature 438, 201 (2005).

[5] Y. Hasegawa, R. Konno, H. Nakano, and M. Kohmoto, Phys. Rev. B 74, 033413 (2006).

[6] V. M. Pereira, A. H. Castro Neto, and N. M. R. Peres, Phys. Rev. B 80, 045401 (2009).

[7] P. Dietl, F. Piéchon, and G. Montambaux, Phys. Rev. Lett. 100, 236405 (2008).

[8] G. Montambaux, F. Piéchon, J.-N. Fuchs, and M. O. Goerbig, Phys. Rev. B 80, 153412 (2009).

[9] P. Sinha, S. Murakami, and S. Basu, Phys. Rev. B 102, 085416 (2020).

[10] J. Kim, S. S. Baik, S. H. Ryu, Y. Sohn, S. Park, B. G. Park, J. Denlinger, Y. Yi, H. J. Choi, and K. S. Kim, Science 349, 723-726 (2015).

[11] S. S. Baik, K. S. Kim, Y. Yi, and H. J. Choi, Nano Lett. 15, 7788 (2015).

[12] Q. Liu, X. Zhang, L. B. Abdalla, A. Fazzio, and A. Zunger, Nano Lett. 15, 1222 (2015).

[13] B. Ghosh, B. Singh, R. Prasad, and A. Agarwal, Phys. Rev. B 94, 205426 (2016).

[14] H. Doh and H. J. Choi, 2D Mater. 4, 025071 (2017).

[15] C. Wang, Q. Xia, Y. Nie, and G. Guo, J. Appl. Phys 117, 124302 (2015).

[16] V. Pardo and W. E. Pickett, Phys. Rev. Lett. 102, 166803 (2009).

[17] S. Banerjee, R. R. P. Singh, V. Pardo, and W. E. Pickett, Phys. Rev. Lett. 103, 016402 (2009).

[18] H. Huang, Z. Liu, H. Zhang, W. Duan, and D. Vanderbilt, Phys. Rev. B 92, 161115(R) (2015).

[19] C. Zhong, Y. Chen, Y. Xie, Y.-Y. Sun and S. Zhang, Phys. Chem. Chem. Phys. 19, 3820 (2017).

[20] S. Tang and M. S. Dresselhaus, Nanoscale 4, 7786 (2012).

[21] H. Zhang, Y. Xie, Z. Zhang, C. Zhong, Y. Li, Z. Chen, and Y. Chen, J. Phys. Chem. Lett. 8, 1707 (2017).

[22] C. Wang, Q. Xia, Y. Nie, M. Rahman, and G. Guo, AIP Adv. 6, 035204 (2016).

[23] Q. Li, P. Ghosh, J. D. Sau, S. Tewari, and S. Das Sarma, Phys. Rev. B 83, 085110 (2011).

[24] F. Zhai, P. Mu, and K. Chang, Phys. Rev. B 83, 195402 (2011).

[25] L. Tarruell, D. Greif, T. Uehlinger, G. Jotzu, and T. Esslinger,
Nature 483, 302 (2012).

[26] B. Real, O. Jamadi, M. Milicevic, N. Pernet, P. St-Jean, T. Ozawa, G. Montambaux, I. Sagnes, A. Lemaltre, L. Le Gratiet, A. Harouri, S. Ravets, J. Bloch, and A. Amo, Phys. Rev. Lett. 125, 186601 (2020).

[27] P. Delplace and G. Montambaux, Phys. Rev. B 82, 035438 (2010).

[28] J. Jang, S. Ahn, and H. Min, 2D Mater. 6, 025029 (2019).

[29] J. P. Carbotte, K. R. Bryenton, and E. J. Nicol, Phys. Rev. B 99, 115406 (2019).

[30] S. Banerjee and W. E. Pickett, Phys. Rev. B 86, 075124 (2012).

[31] F. Zhai, and J. Wang, J. Appl. Phys 116, 063704 (2014).

[32] P. D. Gorman, J. M. Duffy, S. R. Power, and M. S. Ferreira, Phys. Rev. B 90, 125411 (2014).

[33] J. Friedel, Philos. Mag. 43, 153 (1952).

[34] L. Chen, P. Cheng and K. Wu, J. Phys.: Condens. Matter 29, 103001 (2017).

[35] N. Avraham, J. Reiner, A. K.-Nayak, N. Morali, R. Batabyal, B. Yan, and H. Beidenkopf, Adv. Mater. 30, 1707628 (2018).

[36] M. F. Crommie, C. P. Lutz and D. M. Eigler, Nature. 363, 524 (1993).

[37] L. Petersen, P. T. Sprunger, Ph. Hofmann, E. Lægsgaard, B. G. Briner, M. Doering, H.-P. Rust, A. M. Bradshaw, F. Besenbacher, E.W. Plummer, Phys. Rev. B 57, R6858(R) (1998).

[38] J. Xue, J. Sanchez-Yamagishi, K. Watanabe, T. Taniguchi, P. Jarillo-Herrero, and B. J. LeRoy, Phys. Rev. Lett. 108, 016801 (2012).

[39] J. Wang, W. Li, P. Cheng, C. Song, T. Zhang, P. Deng, X. Chen, X. Ma, K. He, J.-F. Jia, Q.-K. Xue, and B.-F. Zhu, Phys. Rev. B 84, 235447 (2011).

[40] R. R. Biswas and A. V. Balatsky, Phys. Rev. B 83, 075439 (2011).

[41] R. R. Biswas and A. V. Balatsky, arXiv:1005.4780.

[42] Q. Liu, X.-L. Qi, and S.-C. Zhang, Phys. Rev. B 85, 125314 (2012).

[43] M. M. Dignam, Phys. Rev. B 50, 2241 (1994).

[44] D. M. Sedrakian, J. Contemp. Phys 45, 118-125 (2010).

[45] Yongjin Jiang, Feng Lu, Feng Zhai, Tony Low, and Jiangping Hu, Phys. Rev. B 84, 205324 (2011).

[46] Y. Korniyenko, Quantum theory of time-dependent transport in graphene (Sweden 2017).

[47] Huaihua Shao, Yiman Liu, Xiaoying Zhou and Guanghui Zhou, Chin. Phys. B 23, 107304 (2014).

[48] J. An, and C. S. Ting, Phys. Rev. B 86, 165313 (2012). 